\title{
Bosonic Realizations of the Colour Heisenberg Lie Algebra
}

\author{
Gunnar SIGURDSSON ${ }^{\dagger}$ and Sergei D SILVESTROV ${ }^{\ddagger}$ \\ $\dagger$ Department of Theoretical Physics, School of Engineering Sciences, Royal Institute \\ of Technology (KTH) - AlbaNova University Center, SE-106 91 Stockholm, Sweden \\ E-mail: gunnsi@kth.se \\ $\ddagger$ Centre for Mathematical Sciences, Lund University, Box 118, SE-221 00 Lund, Sweden \\ E-mail: ssilvest@maths.lth.se
}

This article is part of the Proceedings of the Baltic-Nordic Workshop, Algebra, Geometry and Mathematical Physics which was held in Tallinn, Estonia, during October 2005.

\begin{abstract}
We describe realizations of the colour analogue of the Heisenberg Lie algebra by power series in non-commuting indeterminates satisfying Heisenberg's canonical commutation relations of quantum mechanics. The obtained formulas are used to construct new operator representations of the colour Heisenberg Lie algebra. These representations are shown to be closely connected with some combinatorial identities and functional difference-differential interpolation formulae involving Euler numbers.
\end{abstract}

Key words: Bosonic realizations, Colour Heisenberg Lie algebra, power series, Heisenberg's canonical commutation relations

AMS MSC 2000: 17B75

\section{Introduction}

The main object studied in this article is the unital associative algebra with three generators $A_{1}, A_{2}$ and $A_{3}$ satisfying the defining commutation relations

$$
\begin{aligned}
& A_{1} A_{2}+A_{2} A_{1}=A_{3}, \\
& A_{1} A_{3}+A_{3} A_{1}=0, \\
& A_{2} A_{3}+A_{3} A_{2}=0 .
\end{aligned}
$$

The main goal is to show how $A_{1}, A_{2}$ and $A_{3}$ can be expressed, using elements $A$ and $B$, obeying Heisenberg's canonical commutation relation

$$
A B-B A=I .
$$

The canonical representation of the commutation relation (1.4) is given by choosing $A$ as the usual differentiation operator and $B$ as multiplication by $x$ acting on differentiable 
functions of one real variable $x$, on polynomials in one variable, or on some other suitable linear space of functions invariant under these operators. In quantum mechanics, these operators, when considered on a subspace of a Hilbert space of square integrable functions, are essentially the same as the canonical Heisenberg-Schrödinger observables of momentum and coordinate, differing just by a complex scaling factor. The Heisenberg canonical commutation relation (1.4) is also satisfied by the annihilation and creation operators in a quantum harmonic oscillator.

A complex associative algebra $L$ with generators $A_{1}, A_{2}, A_{3}$ and defining relations (1.1)-(1.3) is called the colour (or graded) analogue of the Heisenberg Lie algebra or, more precisely, of its universal enveloping algebra. The algebra $L$ is a universal enveloping algebra of a three-dimensional $\mathbb{Z}_{2}^{3}$-graded generalized Lie algebra (see Appendix A). When anticommutators in the left-hand side of relations (1.1)-(1.3) are changed into commutators, we indeed have the relations between generators in the universal enveloping algebra of the Heisenberg Lie algebra.

Since the 1970's, generalized (colour) Lie algebras have been an object of constant interest in both mathematics and physics [2,3,6-11,13-19]. Description of representations of these algebras is an important and interesting general problem. It is well known that representations of three-dimensional Lie algebras play an important role in the representation theory of general Lie algebras and groups, both as test examples and building blocks. Similarly, one would expect the same to be true for three-dimensional colour Lie algebras and superalgebras with respect to general colour Lie algebras and superalgebras. The representations of non-isomorphic algebras have different structure. In $[18,19]$, three-dimensional colour Lie algebras are classified in terms of their structure constants, that is in terms of commutation relations between generators. In [11,16], quadratic central elements and involutions on these algebras are calculated. In $[10,17]$, Hilbert space $*$-representations are described for the graded analogues of the Lie algebra $\mathfrak{s l}(2, \mathbb{C})$ and of the Lie algebra of the group of plane motions, two of the non-trivial algebras from the classification. The classification of *-representations in $[10,17]$ is achieved, using the method of dynamical systems based on generalized Mackey imprimitivity systems.

The colour Heisenberg Lie algebra defined by relations (1.1)-(1.3) is another important non-trivial algebra in the classification of three-dimensional colour Lie algebras obtained in $[18,19]$. In this paper we look for representations of this algebra. Here, however, we approach representations in a totally different way than it was done in $[10,17]$. Namely, we are interested in describing those representations which can be obtained as power series in representations of Heisenberg's canonical commutation relations.

In Section 2 we show that, with a natural choice for $A_{1}$ as the first generator of the Heisenberg algebra corresponding to differentiation, there are no non-zero polynomials in Heisenberg generators which can be taken as $A_{2}$ and $A_{3}$ so that the relations (1.1)-(1.3) are satisfied. This means, in particular, that when $A_{1}$ is the differentiation operator, $A_{2}$ and $A_{3}$ cannot be chosen as differential operators of finite order with polynomial coefficients. We prove, however, that it is possible for $A_{2}$ and $A_{3}$ to be power series in the Heisenberg generators with infinitely many non-zero terms, thus in particular making possible the operator representations by the differential operators of infinite order. In Lemma 3, we describe all such formal power series solutions $A_{2}$ and $A_{3}$ for the two relations (1.1)-(1.2). In Theorem 5, we present all formal power series solutions $A_{2}$ and $A_{3}$ satisfying all three relations (1.1)-(1.3). 
By choosing various pairs of operators satisfying the Heisenberg canonical commutation relation (1.4) and substituting them into the power series obeying (1.1)-(1.3), one can find large classes of operator representations of the commutation relations (1.1)-(1.3). Section 3 is exclusively devoted to examples of such representations. Many of these representations, we believe, cannot be reached or classified using classical methods based on dynamical systems approach extending Mackey imprimitivity systems. We think that these operator representations might have significant physical applications. It would be of great interest to investigate spectral, structural and analytical properties of such representations on various spaces.

\section{Bosonic power series realizations}

To fix notation we point out that throughout we let $\mathbb{C}$ denote the field of complex numbers and $\mathbb{N}$ the set of non-negative integers. By $\mathbb{C}[x]$ and $\mathbb{C}[[x]]$ we mean the ring of polynomials and formal power series over $\mathbb{C}$, respectively.

Consider a set $\left\{A_{1}, A_{2}, A_{3}\right\}$ in an associative algebra over $\mathbb{C}$ with unit element $I$ satisfying commutation relations (1.1)-(1.3). Clearly $A_{3}^{2}$ commutes with each element $A_{1}, A_{2}$ and $A_{3}$. Suppose there exists a non-zero scalar $\alpha$ such that $A_{3}^{2}=\alpha^{2} I$. By relations (1.1) and (1.2) we then have

$$
A_{1}\left(A_{2} A_{3}\right)-\left(A_{2} A_{3}\right) A_{1}=A_{3}^{2}=\alpha^{2} I .
$$

Putting $\hat{A}_{2}=\alpha^{-1} A_{2}$ and $\hat{A}_{3}=\alpha^{-1} A_{3}$, we obtain

$$
A_{1}\left(\hat{A}_{2} \hat{A}_{3}\right)-\left(\hat{A}_{2} \hat{A}_{3}\right) A_{1}=I,
$$

showing that $A_{1}$ and the combination $\hat{A}_{2} \hat{A}_{3}$ satisfy the relation (1.4). By the way, this observation implies in particular that the relations (1.1)-(1.3) together with $A_{3}^{2}=\alpha^{2} I, \alpha \neq$ 0 , cannot be satisfied by bounded operators on a Hilbert space or even generally by elements in any unital normed algebra, as this is also the case for the Heisenberg canonical commutation relation (1.4) by the famous Wintner-Wielandt result [12, 20,21].

Assume we consider $A_{1}, A_{2}$ and $A_{3}$ as elements of the Heisenberg algebra $\mathcal{H}_{1}(A, B)=$ $\mathbb{C}\langle A, B\rangle /\langle A B-B A-I\rangle$. Then equation (2.1) suggests that a reasonable Ansatz is to put $A_{1}=A$ and let $A_{2}$ and $A_{3}$ be complex polynomials in $A$ and $B$. Let $P(A, B)$ be an arbitrary polynomial in $A$ and $B$ with complex coefficients. Applying the relation $A B=I+B A, P(A, B)$ can be rewritten as a linear combination of monomials with no $B$ to the right of an $A$. When a polynomial (or a power series) in $A$ and $B$ is written in such a way, we say that it is presented in its $(B, A)$-normal form. In the Heisenberg algebra $\mathcal{H}_{1}(A, B)$, we know that the set of ordered monomials $\left\{B^{j} A^{k} \mid j, k \in \mathbb{N}\right\}$ is linearly independent. This fact allows us to reduce the problem of equality of two polynomials in $A$ and $B$ to checking whether they have the same coefficients when rewritten in the $(B, A)$-normal form.

However, as we will see in Corollary 2, in order to satisfy relations (1.1)-(1.3) it is preferable to use formal power series in $A$ and $B$ instead of polynomials. When computing with power series in non-commuting elements $A$ and $B$ of an associative unital algebra $\mathfrak{A}$, we use the usual addition and multiplication rules of the Magnus algebra of noncommutative formal power series in $A$ and $B$ (see [1]). However, we assume that $A$ and $B$ are not free, 
but satisfy at least the Heisenberg commutation relation as elements in $\mathfrak{A}$. We denote the obtained algebra by $\mathcal{H}_{1}\langle\langle A, B ; \mathfrak{A}\rangle\rangle$. In addition to the subalgebra of $\mathfrak{A}$ generated by $A$ and $B$, consisting of noncommutative polynomials in $A$ and $B$, the algebra $\mathcal{H}_{1}\langle\langle A, B ; \mathfrak{A}\rangle\rangle$ often contains other elements which are infinite noncommutative power series in $A$ and $B$ not belonging to $\mathfrak{A}$. The problem of equality of two elements in $\mathcal{H}_{1}\langle\langle A, B ; \mathfrak{A}\rangle\rangle$ is a very complex matter in itself, deeply connected both to the properties of noncommutative power series and Heisenberg's relation and to the structure of the algebra $\mathfrak{A}$, and properties of $A$ and $B$ in $\mathfrak{A}$. We say that an element of $\mathcal{H}_{1}\langle\langle A, B ; \mathfrak{A}\rangle\rangle$ is in the $(B, A)$-normal form (respectively $(A, B)$-normal form) if it is a noncommutative power series built of only ordered monomials $\left\{B^{j} A^{k} \mid j, k \in \mathbb{N}\right\}$ (respectively $\left\{A^{j} B^{k} \mid j, k \in \mathbb{N}\right\}$ ). In order to be able to enjoy the equality properties in a similar way with formal power series as in the polynomial case, we assume throughout this article that two formal power series in $A$ and $B$, written in the $(B, A)$-normal form (or respectively in the $(A, B)$-normal form), are equal if and only if their coefficients are the same and in particular such a series is zero if and only if all coefficients are zero. This important equality assumption is actually an assumption on $\mathcal{H}_{1}\langle\langle A, B ; \mathfrak{A}\rangle\rangle$, on the algebra $\mathfrak{A}$ as well as on $A$ and $B$ as elements in $\mathfrak{A}$. In the particular case of polynomials in $A$ and $B$, that is for the subalgebra of $\mathfrak{A}$ generated by $A$ and $B$, the assumption yields the same property as in $\mathcal{H}_{1}(A, B)$, namely that $\left\{B^{j} A^{k} \mid j, k \in \mathbb{N}\right\}$ and $\left\{A^{j} B^{k} \mid j, k \in \mathbb{N}\right\}$ are linearly independent as subsets of $\mathfrak{A}$.

With the assumption above we may claim the equality of two elements of $\mathcal{H}_{1}\langle\langle A, B ; \mathfrak{A}\rangle\rangle$ if they are equal to the same element in $(B, A)$-normal form (or in $(A, B)$-normal form). However, it is important to observe that $\mathcal{H}_{1}\langle\langle A, B ; \mathfrak{A}\rangle\rangle$ may well contain elements which cannot be represented on $(B, A)$-normal form, or $(A, B)$-normal form or even on either of them. We refer to [4] for further discussion on power series extensions of the Heisenberg algebra, Diamond lemma and normal forms. The reordering relations (see for instance [5])

$$
\begin{aligned}
A^{i} B^{j} & =\sum_{\nu=0}^{\min (i, j)} \nu !\left(\begin{array}{l}
i \\
\nu
\end{array}\right)\left(\begin{array}{l}
j \\
\nu
\end{array}\right) B^{j-\nu} A^{i-\nu}, \\
B^{i} A^{j} & =\sum_{\nu=0}^{\min (i, j)}(-1)^{\nu} \nu !\left(\begin{array}{l}
i \\
\nu
\end{array}\right)\left(\begin{array}{l}
j \\
\nu
\end{array}\right) A^{j-\nu} B^{i-\nu}, \\
f(A) B & =B f(A)+f^{\prime}(A), \quad A g(B)=g(B) A+g^{\prime}(B)
\end{aligned}
$$

are valid for all non-negative $i$ and $j$, as long as $A$ and $B$ satisfy relation (1.4) and $f(A)$ and $g(B)$ are polynomials (or power series) in $A$ and $B$, respectively. Here $f^{\prime}(A)$ and $g^{\prime}(B)$ denote (formal) derivatives, obtained by termwise differentiation of the polynomials (power series). These relations can be used to prove the following statement, showing that two non-trivial polynomials in $A$ and $B$ cannot anticommute if the condition of linear independence of ordered monomials is satisfied. Since this statement is important for this article and we do not know any explicit reference for this fact, we include it here with a short proof for completeness of exposition.

Proposition 1. Assume $A$ and $B$ are two elements in a complex associative algebra with unity $I$ satisfying the Heisenberg canonical commutation relation $A B-B A=I$. If $P, Q \in \mathcal{H}_{1}(A, B)$ satisfy the relation $P Q+Q P=0$, then it follows that at least one of these polynomials must vanish. 
Proof. Since any polynomial in $A$ and $B$ can be written in the $(B, A)$-normal form, we can assume that

$$
P=\sum_{j=0}^{K} \sum_{k=0}^{L} \alpha_{j k} B^{j} A^{k}, \quad Q=\sum_{j=0}^{M} \sum_{k=0}^{N} \beta_{j k} B^{j} A^{k}
$$

for some $K, L, M, N \in \mathbb{N}$ and $\alpha_{j k}, \beta_{j k} \in \mathbb{C}$. Each of the sets $\left\{\alpha_{K k} \mid k=1, \ldots, L\right\}$ and $\left\{\beta_{M k} \mid k=1, \ldots, N\right\}$ can be supposed to contain at least one non-zero element, so that in variable $B$ the polynomials $P$ and $Q$ have degree $K$ and $M$, respectively. Let $p(A)$ and $q(A)$ be the polynomials in $A$ given by $p(A)=\sum_{k=0}^{L} \alpha_{K k} A^{k}$ and $q(A)=\sum_{k=0}^{N} \beta_{M k} A^{k}$. Applying reordering relation (2.2), we obtain

$$
P Q+Q P=2 B^{K+M} p(A) q(A)+\sum_{j=0}^{K+M-1} \sum_{k=0}^{L+N} \gamma_{j k} B^{j} A^{k},
$$

for some set of complex constants $\gamma_{j k}$. If $P Q+Q P=0$ then, by the linear independence of the ordered monomials $\left\{B^{j} A^{k} \mid j, k \in \mathbb{N}\right\}$, this implies that $p(A) q(A)=0$, and hence, since the set of complex polynomials in $A$ has no zero divisors, that at least one of the factors $p(A)$ or $q(A)$ must vanish. This contradicts our assumption that $P$ and $Q$ are polynomials of $B$-degree $K$ and $M$, respectively.

Corollary 2. Assume $A_{1}=A$ and let $A_{2}$ and $A_{3}$ be polynomials in the Heisenberg algebra $\mathcal{H}_{1}(A, B)$. Then it follows that the commutation relations

$$
A_{1} A_{2}+A_{2} A_{1}=A_{3}, \quad A_{1} A_{3}+A_{3} A_{1}=0
$$

can only be satisfied if $A_{2}=A_{3}=0$.

Proof. By the second relation we have $A A_{3}+A_{3} A=0$. Applying Proposition 1 we obtain $A_{3}=0$. Combining this with the first relation yields $A A_{2}+A_{2} A=0$, and hence, by Proposition $1, A_{2}=0$.

Guided by this result concerning elements of the Heisenberg algebra, we see that one is forced to work with series in $A$ and $B$ with infinitely many non-zero terms, in order to be able to find non-trivial realizations of the commutation relations $A_{1} A_{2}+A_{2} A_{1}=A_{3}$ and $A_{1} A_{3}+A_{3} A_{1}=0$ in terms of the Heisenberg generators $A$ and $B$.

Lemma 3. Let $A_{1}=A$ and assume that $A_{2}$ and $A_{3}$ are elements of the algebra $\mathcal{H}_{1}\langle\langle A, B ; \mathfrak{A}\rangle\rangle$ written in the $(B, A)$-normal form, i.e.,

$$
A_{2}=\sum_{j=0}^{\infty} \sum_{k=0}^{\infty} a_{j k} B^{j} A^{k}, \quad A_{3}=\sum_{j=0}^{\infty} \sum_{k=0}^{\infty} \tilde{a}_{j k} B^{j} A^{k}, \quad a_{j k}, \tilde{a}_{j k} \in \mathbb{C} .
$$

Then $A_{1}, A_{2}$ and $A_{3}$ satisfy the commutation relations

$$
A_{1} A_{2}+A_{2} A_{1}=A_{3}, \quad A_{1} A_{3}+A_{3} A_{1}=0
$$

if and only if

$$
A_{2}=T(B, A) V(A)+B T(B, A) W(A), \quad A_{3}=T(B, A) W(A),
$$

where $T(B, A)=\sum_{k=0}^{\infty} \frac{(-2)^{k}}{k !} B^{k} A^{k}$ and $V(A), W(A) \in \mathbb{C}[[A]]$. 
Proof. We look for the general solution to the equation $A Z+Z A=0$ in the form

$$
Z=\sum_{j=0}^{\infty} \sum_{k=0}^{\infty} c_{j k} B^{j} A^{k}, \quad c_{j k} \in \mathbb{C} .
$$

We have, using the relation $A B^{n}=B^{n} A+n B^{n-1}$ which is a special case of (2.2),

$$
\begin{aligned}
A Z+Z A & =\sum_{j=0}^{\infty} \sum_{k=0}^{\infty} c_{j k} A B^{j} A^{k}+\sum_{j=0}^{\infty} \sum_{k=0}^{\infty} c_{j k} B^{j} A^{k+1} \\
& =\sum_{j=0}^{\infty} \sum_{k=0}^{\infty} 2 c_{j k} B^{j} A^{k+1}+\sum_{j=1}^{\infty} \sum_{k=0}^{\infty} j c_{j k} B^{j-1} A^{k} \\
& =\sum_{j=0}^{\infty} \sum_{k=0}^{\infty} 2 c_{j k} B^{j} A^{k+1}+\sum_{j=0}^{\infty} \sum_{k=-1}^{\infty}(j+1) c_{j+1, k+1} B^{j} A^{k+1} \\
& =\sum_{j=0}^{\infty}(j+1) c_{j+1,0} B^{j}+\sum_{j=0}^{\infty} \sum_{k=0}^{\infty}\left[2 c_{j k}+(j+1) c_{j+1, k+1}\right] B^{j} A^{k+1} .
\end{aligned}
$$

The series is here given in its $(B, A)$-normal form. If now $A Z+Z A=0$, then all coefficients must be equal to zero giving rise to the following recurrence relation with initial conditions

$$
(j+1) c_{j+1, k+1}+2 c_{j k}=0, \quad c_{j+1,0}=0, \quad j, k \in \mathbb{N} .
$$

As a consequence of equations (2.5) we have immediately $c_{i+1+j, j}=0$, for $i, j \in \mathbb{N}$. In order to find the remaining elements of the sequence, we now put $l=k-j$, obtaining

$$
(j+1) c_{j+1, j+l+1}+2 c_{j, j+l}=0,
$$

where $j, l \in \mathbb{N}$. For every fixed $l$ we have a two-term linear recursion with respect to $j$. The solution is readily found to be

$$
c_{j+1, j+l+1}=\frac{(-2)^{j+1}}{(j+1) !} c_{0 l}
$$

where $j, l \in \mathbb{N}$ and $c_{0 l}$ can be chosen as arbitrary complex numbers. Setting $c_{0 l}=z_{l}$ for all $l$ the solution to the problem (2.5) can now be written

$$
c_{j, j+l}=\frac{(-2)^{j}}{j !} z_{l}, \quad c_{j+1+l, l}=0,
$$

where $j, l \in \mathbb{N}$ and $\left(z_{i}\right)_{i=0}^{\infty}$ is an arbitrary sequence of complex numbers. By virtue of the solution (2.7), we may write

$$
Z=\sum_{j=0}^{\infty} \sum_{k=0}^{\infty} c_{j k} B^{j} A^{k}=\sum_{j=0}^{\infty} \sum_{l=0}^{\infty} c_{j, j+l} B^{j} A^{j+l}=\sum_{j=0}^{\infty} \sum_{l=0}^{\infty} \frac{(-2)^{j}}{j !} z_{l} B^{j} A^{j+l}
$$

Alternatively, we can express the general solution $Z$ by separating the formal summations as

$$
Z=\sum_{k=0}^{\infty} \frac{(-2)^{k}}{k !} B^{k} A^{k} \sum_{l=0}^{\infty} z_{l} A^{l}
$$


A system consisting of the two relations $A Y+Y A=Z, A Z+Z A=0$ has a general solution $(Y, Z)$, where $Y=Y_{h}+Y_{p}$ with $Y_{p}$ taken as a particular series satisfying the inhomogeneous relation $A Y_{p}+Y_{p} A=Z$, and $Y_{h}$ being the general solution to the homogeneous equation $A Y+Y A=0$. We know from above (the problem for $Z$ ) that

$$
Y_{h}=\sum_{k=0}^{\infty} \frac{(-2)^{k}}{k !} B^{k} A^{k} \sum_{l=0}^{\infty} y_{l} A^{l},
$$

where $\left(y_{i}\right)_{i=0}^{\infty}$ is an arbitrary sequence of complex numbers. Moreover, we can choose

$$
Y_{p}=B Z=B \sum_{k=0}^{\infty} \frac{(-2)^{k}}{k !} B^{k} A^{k} \sum_{l=0}^{\infty} z_{l} A^{l} .
$$

since $A B Z+B Z A=A B Z-B A Z=Z$ by (1.4). This proves the lemma.

In Lemma 3 , the formal series $A_{2}$ and $A_{3}$ are expressed in the $(B, A)$-normal form. This is a natural ordering when we think of $A$ as the usual differentiation operator $\partial$ and $B$ as a multiplication operator $M$ acting on differentiable functions on the real line, given by $\partial f=f^{\prime}$ and $M f(t)=t f(t)$. In other situations, it may be more appropriate to consider the reversed order.

Taking $A_{2}$ and $A_{3}$ in Lemma 3 to be in the $(A, B)$-normal form, but keeping $A_{1}=A$, the general solution will be changed to the following form

$$
A_{2}=V(A) U(A, B)-W(A) U(A, B) B, \quad A_{3}=W(A) U(A, B),
$$

where $U(A, B)=\sum_{k=0}^{\infty} \frac{2^{k}}{k !} A^{k} B^{k}$. The proof goes along the same lines as the proof of Lemma 3.

In the following lemma, we formulate some important basic relations satisfied by the series $T(B, A)$ and $U(A, B)$. These relations are very useful in many computations and examples. In particular they are instrumental in the proof of our main result, Theorem 5.

Lemma 4. Suppose $A$ and $B$ are two elements in a complex associative algebra with unit element $I$ satisfying the Heisenberg canonical commutation relation $A B-B A=I$. Let $f(A)$ and $g(B)$ be formal power series in $A$ and $B$, respectively. If $T(B, A)$ and $U(A, B)$ are defined by

$$
T(B, A)=\sum_{k=0}^{\infty} \frac{(-2)^{k}}{k !} B^{k} A^{k}, \quad U(A, B)=\sum_{k=0}^{\infty} \frac{2^{k}}{k !} A^{k} B^{k},
$$

then the following relations hold true

(a) $\begin{aligned} A T(B, A)+T(B, A) A=0, & T(B, A) B+B T(B, A)=0, \\ A U(A, B)+U(A, B) A=0, & U(A, B) B+B U(A, B)=0 .\end{aligned}$

(b) $f(A) T(B, A)=T(B, A) f(-A), \quad T(B, A) g(B)=g(-B) T(B, A)$, $U(A, B) f(A)=f(-A) U(A, B), \quad g(B) U(A, B)=U(A, B) g(-B)$.

(c) $T(B, A)^{2}=U(A, B)^{2}=I$. 
Proof. a) This is an easy verification having in mind the commutation relations (2.4).

b) Follows immediately from the relations in a) by mathematical induction.

c) Using the definition (2.11) and reordering relation (2.2), we have

$$
\begin{aligned}
T(B, A) T(B, A) & =\sum_{k=0}^{\infty} \frac{(-2)^{k}}{k !} B^{k} A^{k} \sum_{m=0}^{\infty} \frac{(-2)^{m}}{m !} B^{m} A^{m} \\
& =\sum_{k=0}^{\infty} \sum_{m=0}^{\infty} \frac{(-2)^{k+m}}{k ! m !} B^{k} A^{k} B^{m} A^{m} \\
& =\sum_{k=0}^{\infty} \sum_{m=0}^{\infty} \sum_{\nu=0}^{\min (k, m)} \frac{(-2)^{k+m}}{k ! m !} \nu !\left(\begin{array}{c}
k \\
\nu
\end{array}\right)\left(\begin{array}{c}
m \\
\nu
\end{array}\right) B^{k+m-\nu} A^{k+m-\nu} \\
& =\sum_{k=0}^{\infty} \sum_{m=0}^{\infty} \sum_{\nu=0}^{\min (k, m)} \frac{(-2)^{k+m}}{k !(m-\nu) !}\left(\begin{array}{c}
k \\
\nu
\end{array}\right) B^{k+m-\nu} A^{k+m-\nu} .
\end{aligned}
$$

Introducing a new summation index $r=k+m-\nu$, this can be expressed as

$$
T(B, A) T(B, A)=\sum_{r=0}^{\infty} d_{r} B^{r} A^{r}
$$

where

$$
\begin{aligned}
d_{r} & =\sum_{k=0}^{r} \sum_{m=r-k}^{r} \frac{(-2)^{k+m}}{k !(r-k) !}\left(\begin{array}{c}
k \\
k+m-r
\end{array}\right)=(-2)^{r} \sum_{k=0}^{r} \frac{1}{k !(r-k) !} \sum_{\nu=0}^{k}(-2)^{\nu}\left(\begin{array}{l}
k \\
\nu
\end{array}\right) \\
& =\frac{(-2)^{r}}{r !} \sum_{k=0}^{r}(-1)^{k}\left(\begin{array}{l}
r \\
k
\end{array}\right)=\frac{(-2)^{r}}{r !} \delta_{r 0}=\delta_{r 0} .
\end{aligned}
$$

Hence, $T(B, A)^{2}=\sum_{r=0}^{\infty} d_{r} B^{r} A^{r}=\sum_{r=0}^{\infty} \delta_{r 0} B^{r} A^{r}=I$. The proof of $U(A, B)^{2}=I$ is analogous and uses the reordering relation (2.3).

Remark 1. The series $T(B, A)$ can be seen as an abstract generalization of the parity operator $f(x) \mapsto f(-x)$. The usual parity operator is obtained in the special case of canonical representation of the Heisenberg relation (1.4) when $A=\partial: f(x) \mapsto f^{\prime}(x)$ is differentiation and $B=M: f(x) \mapsto x f(x)$ is multiplication operator acting on functions on $\mathbb{R}$. This is proved in the beginning of Section 3.

Now, let $A_{2}$ and $A_{3}$ be given in the $(B, A)$-normal form as

$$
A_{2}=T(B, A) V(A)+B T(B, A) W(A), \quad A_{3}=T(B, A) W(A),
$$

where $V(A), W(A) \in \mathbb{C}[[A]]$ (cf. Lemma 3). Applying the rules of Lemma 4 yields

$$
A_{2} A_{3}=V(-A) W(A)+B W(-A) W(A), \quad A_{3}^{2}=W(-A) W(A) .
$$

On the other hand, if $A_{2}$ and $A_{3}$ are expressed in the $(A, B)$-normal form as

$$
A_{2}=V(A) U(A, B)-W(A) U(A, B) B, \quad A_{3}=W(A) U(A, B),
$$


then Lemma 4 implies that

$$
\begin{aligned}
& A_{2} A_{3}=V(A) W(-A)+W(A) W(-A) B+W(A) W^{\prime}(-A), \\
& A_{3} A_{3}=W(A) W(-A),
\end{aligned}
$$

where $W^{\prime}(A)$ is the formal derivative of $W(A)$, obtained by termwise differentiation of the power series.

In the following theorem, being the main result of this article, we give the general solution to the problem with all three relations (1.1)-(1.3). In the formulation, the exponential generating function $E(t)$ of the so-called Euler numbers is used. Recall that one defines a sequence of polynomials $E_{k}(x)$, called the Euler polynomials, by specifying their exponential generating function as

$$
\frac{2 e^{x t}}{e^{t}+1}=\sum_{k=0}^{\infty} E_{k}(x) \frac{t^{k}}{k !}, \quad|t|<\pi .
$$

The four polynomials of lowest degree are

$$
E_{0}(x)=1, \quad E_{1}(x)=x-\frac{1}{2}, \quad E_{2}(x)=x^{2}-x, \quad E_{3}(x)=x^{3}-\frac{3}{2} x^{2}+\frac{1}{4} .
$$

The Euler numbers $E_{k}$ are then defined as the integers $E_{k}=2^{k} E_{k}\left(\frac{1}{2}\right)$. It follows that $E_{0}=1, E_{1}=0, E_{2}=-1, E_{3}=0$ and generally for $k \geq 0$

$$
E_{2 k+1}=0, \quad E_{2 k}=(-1)^{k} \frac{(2 k) ! 2^{2 k+2}}{\pi^{2 k+1}} \sum_{\nu=0}^{\infty}(-1)^{\nu}(2 \nu+1)^{-2 k-1} .
$$

The Euler numbers have an exponential generating function obtained by setting $x=1 / 2$ and replacing $t$ by $2 t$ in the exponential generating function of the Euler polynomials

$$
E(t)=\frac{2 e^{t}}{e^{2 t}+1}=\sum_{k=0}^{\infty} E_{k} \frac{t^{k}}{k !}
$$

Theorem 5. Suppose that $A_{1}, A_{2}$ and $A_{3}$ are elements of the algebra $\mathcal{H}_{1}\langle\langle A, B ; \mathfrak{A}\rangle\rangle$ such that $A_{1}=A$ and $A_{2}, A_{3}$ are formal power series in the $(B, A)$-normal form given as

$$
A_{2}=\sum_{j=0}^{\infty} \sum_{k=0}^{\infty} a_{j k} B^{j} A^{k}, \quad A_{3}=\sum_{j=0}^{\infty} \sum_{k=0}^{\infty} \tilde{a}_{j k} B^{j} A^{k}, \quad a_{j k}, \tilde{a}_{j k} \in \mathbb{C} .
$$

Then $A_{1}, A_{2}$ and $A_{3}$ satisfy the commutation relations

$$
A_{1} A_{2}+A_{2} A_{1}=A_{3}, \quad A_{1} A_{3}+A_{3} A_{1}=0, \quad A_{2} A_{3}+A_{3} A_{2}=0,
$$

if and only if either $A_{3}=0$ and $A_{2}=T(B, A) V(A)$, where $T(B, A)=\sum_{k=0}^{\infty} \frac{(-2)^{k}}{k !} B^{k} A^{k}$ and $V(A) \in \mathbb{C}[[A]]$, or

$$
\begin{aligned}
& A_{2}=c T(B, A) E(\varphi(A))\left[\mathrm{e}^{\varphi(A)} \psi(A)-\frac{1}{2} \varphi^{\prime}(A)\right]+c B T(B, A) \mathrm{e}^{\varphi(A)}, \\
& A_{3}=c T(B, A) \mathrm{e}^{\varphi(A)},
\end{aligned}
$$

where $c$ is a non-zero complex constant, $E(t)$ is the exponential generating function of the Euler numbers and both $\varphi(A)$ and $\psi(A)$ are some odd power series in $\mathbb{C}[[A]]$. 
Proof. By Lemma 3 we have, when considering only relations (1.1)-(1.2), a general solution given by $A_{1}=A$ and equations (2.12). In the present case, $A_{2}$ and $A_{3}$ are supposed to satisfy the additional condition $A_{2} A_{3}+A_{3} A_{2}=0$. Inserting the expressions (2.12) and applying the rules of Lemma 4 , yields

$$
\begin{aligned}
A_{3} A_{2} & =T(B, A) W(A) T(B, A) V(A)+T(B, A) W(A) B T(B, A) W(A) \\
& =T(B, A) T(B, A) W(-A) V(A)-T(B, A) W(A) T(B, A) B W(A) \\
& =W(-A) V(A)-T(B, A) T(B, A) W(-A) B W(A) \\
& =W(-A) V(A)-\left[B W(-A)-W^{\prime}(-A)\right] W(A) \\
& =W(-A) V(A)-B W(-A) W(A)+W^{\prime}(-A) W(A) .
\end{aligned}
$$

Together with the first equation in (2.13) this implies

$$
A_{2} A_{3}+A_{3} A_{2}=V(-A) W(A)+W(-A) V(A)+W^{\prime}(-A) W(A)=0,
$$

which is a functional-differential equation for $V$ and $W$. Since $V$ and $W$ are formal power series, we also have the equation

$$
V(A) W(-A)+W(A) V(-A)+W^{\prime}(A) W(-A)=0 .
$$

Let $V(A)=\sum_{l=0}^{\infty} v_{l} A^{l}$ and $W(A)=\sum_{l=0}^{\infty} w_{l} A^{l}$, where $v_{l}, w_{l} \in \mathbb{C}$. Subtracting equation (2.18) from equation (2.19) yields

$$
W^{\prime}(A) W(-A)-W^{\prime}(-A) W(A)=0 .
$$

Integrating (2.20) by use of Leibniz rule and noting that $W(0)=w_{0}$, we have

$$
W(A) W(-A)=w_{0}^{2} 1 .
$$

First we consider the case when $w_{0}=0$. Knowing that the set of formal power series constitutes an integral domain, it follows from the equation $W(A) W(-A)=0$ that $W(A)=0$. Taking $W(A)$ to be zero in (2.18), implies that there is no equation left for $V(A)$, i.e. $V(A)$ can be chosen arbitrarily in the expression (2.12) for $A_{2}$, proving the first case in the conclusion of the theorem.

Assuming that $w_{0} \neq 0$, we can divide both sides of equation $(2.21)$ by the non-zero constant $w_{0}^{2}$, obtaining the simple equation $g(A) g(-A)=1$, where $g(A)=W(A) / w_{0}$. Taking the logarithm of both sides, it follows that $\log g(A)$ has to be an odd power series expression since $\log g(A)+\log g(-A)=0$. Let $\varphi(A)=\log g(A)$ and we have $W(A)=$ $w_{0} g(A)=w_{0} \exp (\varphi(A))$, which is the general solution to the functional equation (2.21), $\varphi(A)$ being any odd formal power series with complex coefficients. Substituting for $W(A)$ into equation (2.19) yields

$$
V(A) w_{0} \exp (\varphi(-A))+w_{0} \exp (\varphi(A)) V(-A)+w_{0}^{2} \varphi^{\prime}(A)=0 .
$$

This can be written as

$$
\begin{aligned}
& V(A)[\cosh \varphi(A)-\sinh \varphi(A)]+V(-A)[\cosh \varphi(A)+\sinh \varphi(A)]+w_{0} \varphi^{\prime}(A)=0, \\
& {[V(A)+V(-A)] \cosh \varphi(A)=[V(A)-V(-A)] \sinh \varphi(A)-w_{0} \varphi^{\prime}(A),}
\end{aligned}
$$


and we have

$$
2 V(A) \cosh \varphi(A)=[V(A)-V(-A)] \exp (\varphi(A))-w_{0} \varphi^{\prime}(A) .
$$

Denoting the odd part of $V(A)$ by $V_{1}(A)$, we obtain

$$
V(A) \cosh \varphi(A)=V_{1}(A) \exp (\varphi(A))-\frac{w_{0}}{2} \varphi^{\prime}(A) .
$$

The power series $E(X)$ defined by (2.17) is the inverse to the formal power series given by $\cosh (X)$, in the sense that $E(X) \cosh (X)=\cosh (X) E(X)=1$. Multiplying both sides of equation (2.22) by $E(\varphi(A))$ yields $V(A)=E(\varphi(A)) V_{1}(A) \exp (\varphi(A))-\frac{w_{0}}{2} E(\varphi(A)) \varphi^{\prime}(A)$. We have an expression for $V(A)$ in terms of the odd power series $\varphi(A)$ and the odd part $V_{1}(A)$ of $V(A)$. Here $V_{1}(A)$ can be chosen arbitrarily from the set of formal odd power series with coefficients from $\mathbb{C}$. Writing this as $V_{1}(A)=w_{0} \psi(A)$ with $w_{0}$ and $\psi(A)$ arbitrary, we have

$$
V(A)=w_{0} E(\varphi(A)) \exp (\varphi(A)) \psi(A)-\frac{w_{0}}{2} E(\varphi(A)) \varphi^{\prime}(A) .
$$

where $\varphi(A)$ and $\psi(A)$ are arbitrary odd formal power series with complex coefficients.

As was the case for Lemma 3, we can certainly formulate a version of Theorem 5 , where $A_{1}=A$ and the formal series $A_{2}$ and $A_{3}$ are taken to be in the $(A, B)$-normal form. In order for $A_{2}$ and $A_{3}$ to satisfy the relation $A_{2} A_{3}+A_{3} A_{2}=0$, when given by the equations (2.14), one has to take either $W(A)=0$ (with $V(A)$ arbitrary in $\mathbb{C}[[A]]$ ) or

$$
V(A)=c E(\varphi(A))\left[\mathrm{e}^{\varphi(A)} \psi(A)-\frac{1}{2} \varphi^{\prime}(A)\right], \quad W(A)=c \mathrm{e}^{\varphi(A)},
$$

where $c \in \mathbb{C}, c \neq 0$, meaning that

$$
\begin{aligned}
& A_{2}=c E(\varphi(A))\left[\mathrm{e}^{\varphi(A)} \psi(A)-\frac{1}{2} \varphi^{\prime}(A)\right] U(A, B)+c \mathrm{e}^{\varphi(A)} U(A, B) B, \\
& A_{3}=c \mathrm{e}^{\varphi(A)} U(A, B) .
\end{aligned}
$$

This can be proved in a similar way with Theorem 5 .

The relatively simple expressions obtained for $A_{2} A_{3}$ and $A_{3}^{2}$ in equations (2.13), (2.15) and (2.16), being essentially pure formal series in $A$, can now be rewritten using $V(A)$ and $W(A)$ as given by equations $(2.24)$. In the $(B, A)$-normal case we have

$$
A_{2} A_{3}=c B-c E(\varphi(A))\left[\psi(A)+\frac{1}{2} \mathrm{e}^{\varphi(A)} \varphi^{\prime}(A)\right], \quad A_{3}^{2}=c I,
$$

whereas the $(A, B)$-normal form leads to the expressions

$$
A_{2} A_{3}=c B+c \varphi^{\prime}(A)+c E(\varphi(A))\left[\psi(A)-\frac{1}{2} e^{-\varphi(A)} \varphi^{\prime}(A)\right], \quad A_{3}^{2}=c I .
$$

The Heisenberg canonical commutation relation (1.4) can be rewritten in the form $B(-A)-(-A) B=I$. Hence, by defining $\tilde{A}=B$ and $\tilde{B}=-A$, we obtain a new pair of generators satisfying $\tilde{A} \tilde{B}-\tilde{B} \tilde{A}=I$. In general, defining $\tilde{A}$ and $\tilde{B}$ as the complex linear combinations

$$
\tilde{A}=c_{11} A+c_{12} B, \quad \tilde{B}=c_{21} A+c_{22} B
$$


where the coefficient $2 \times 2$ matrix $C=\left(c_{i j}\right)$ has complex entries, new elements $\tilde{A}$ and $\tilde{B}$ will satisfy the commutation relation $\tilde{A} \tilde{B}-\tilde{B} \tilde{A}=I$ if and only if $\operatorname{det} C=1$, i.e. $C \in S L(2, \mathbb{C})$. Consider the special case where $\tilde{A}=B$ and $\tilde{B}=-A$. We can apply Lemma 3 and Theorem 5 , as well as their $(A, B)$-normal form versions described above, on the new set of generators $\tilde{A}$ and $\tilde{B}$. Observing that $T(-A, B)=U(A, B)$ and $U(B,-A)=T(B, A)$ the resulting general solutions are

$$
\begin{array}{lll}
A_{1}=B, & A_{2}=U(A, B) V(B)-A U(A, B) W(B), & A_{3}=U(A, B) W(B), \\
A_{1}=B, & A_{2}=V(B) T(B, A)+W(B) T(B, A) A, & A_{3}=W(B) T(B, A) .
\end{array}
$$

Let $V(t)$ and $W(t)$ be arbitrary formal power series in $\mathbb{C}[[t]]$. Then each of the four suggested solutions (2.12), (2.14), (2.25) and (2.26), with $A_{1}=A$ in the first two cases, will satisfy the pair of commutation relations

$$
A_{1} A_{2}+A_{2} A_{1}=A_{3}, \quad A_{1} A_{3}+A_{3} A_{1}=0 .
$$

If we want to have a triple $\left(A_{1}, A_{2}, A_{3}\right)$ that satisfies all three relations

$$
A_{1} A_{2}+A_{2} A_{1}=A_{3}, \quad A_{1} A_{3}+A_{3} A_{1}=0, \quad A_{2} A_{3}+A_{3} A_{2}=0,
$$

then there will be restrictions on the formal series $V(t)$ and $W(t)$. Note that all free parameters are given by the complex coefficient sequences $\left(v_{i}\right)_{i=0}^{\infty}$ and $\left(w_{i}\right)_{i=0}^{\infty}$ in the formal expressions $V(t)=\sum_{k=0}^{\infty} v_{k} t^{k}$ and $W(t)=\sum_{k=0}^{\infty} w_{k} t^{k}$, whereas both $T(B, A)$ and $U(A, B)$ are fixed power series. The three relations are satisfied if either $W(t)=0$ and $V(t)$ is arbitrary (the rather trivial case which essentially is contained in part b) of Lemma 4) or

$$
V(t)=c E(\varphi(t))\left[\mathrm{e}^{\varphi(t)} \psi(t)-\frac{1}{2} \varphi^{\prime}(t)\right], \quad W(t)=c \mathrm{e}^{\varphi(t)},
$$

where $c$ is a non-zero complex constant, $E(t)$ is the exponential generating function of the Euler numbers $E_{k}$ and both $\varphi(t)$ and $\psi(t)$ are odd formal power series in $t$ having complex coefficients. Here $\varphi^{\prime}(t)$ denotes the formal derivative of $\varphi(t)$.

Remark 2. Note that exchange of $A_{1}$ and $A_{2}$ does not change the commutation relations (1.1)-(1.3). So, by exchanging $A_{1}$ and $A_{2}$ in all statements of the article, we obtain other expressions for $A_{1}$ and $A_{2}$ in terms of the Heisenberg generators.

\section{Some particular bosonic representations}

In this section we will describe some non-trivial particular representations of the colour Heisenberg Lie algebra defined by the commutation relations (1.1)-(1.3). All examples are based on the general statement in Theorem 5 and correspond (except in Example 1) to simple specific choices of the odd formal power series $\varphi(A)$ and $\psi(A)$.

As a concrete example of elements satisfying the Heisenberg commutation relation (1.4), we can consider the differentiation and multiplication operators $\partial$ and $M$ defined on the linear space $\mathbb{C}[x]$ of polynomials. If $f(x)=\sum_{k=0}^{n} f_{k} x^{k}$, then by definition

$$
(\partial f)(x)=\sum_{k=1}^{n} f_{k} k x^{k-1}, \quad(M f)(x)=x f(x)=\sum_{k=0}^{n} f_{k} x^{k+1}
$$


and we have the well-known relation $\partial M-M \partial=I$. As a basis in $\mathbb{C}[x]$ we can take the set of monomials $\left\{1, x, x^{2}, \ldots, x^{n}, x^{n+1}, \ldots\right\}$. Acting on an arbitrary basis vector $x^{n}$, we find

$$
\begin{aligned}
& T(M, \partial)\left(x^{n}\right)=\sum_{k=0}^{\infty} \frac{(-2)^{k}}{k !} M^{k} \partial^{k} x^{n}=\sum_{k=0}^{n} \frac{(-2)^{k}}{k !} x^{k} \frac{n !}{(n-k) !} x^{n-k}=(-x)^{n}, \\
& U(\partial, M)\left(x^{n}\right)=\sum_{j=0}^{\infty} \frac{2^{j}}{j !} \partial^{j} M^{j} x^{n}=\sum_{j=0}^{\infty} \frac{2^{j}}{j !} \partial^{j} x^{n+j}=\sum_{j=0}^{\infty} \frac{2^{j}}{j !} \frac{(n+j) !}{n !} x^{n}=\infty \cdot x^{n} .
\end{aligned}
$$

Thus $T(M, \partial)$ acts as the parity operator, while $U(\partial, M)$ is not well-defined on $\mathbb{C}[x]$. In fact, if $g$ is an analytic function on $\mathbb{R}$, then we have by Taylor's Theorem $\mathrm{e}^{\partial} g(x)=g(x+1)$ and

$$
\begin{aligned}
T(M, \partial) g(x) & =\sum_{k=0}^{\infty} \frac{(-2)^{k}}{k !} M^{k} \partial^{k} g(x)=\sum_{k=0}^{\infty} \frac{(-2)^{k}}{k !} x^{k} g^{(k)}(x) \\
& =\sum_{k=0}^{\infty} \frac{g^{(k)}(x)}{k !}(-2 x)^{k}=g(x-2 x)=g(-x) .
\end{aligned}
$$

Example 1. We consider the operators $A=\partial$ and $B=M$, defined on the linear space $\mathbb{C}[x]$. The general solution given by equations $(2.26)$ and $(2.27)$ is

$$
\begin{aligned}
& A_{1}=M, \quad A_{3}=c \mathrm{e}^{\varphi(M)} T(M, \partial), \\
& A_{2}=c E(\varphi(M))\left[\mathrm{e}^{\varphi(M)} \psi(M)-\frac{1}{2} \varphi^{\prime}(M)\right] T(M, \partial)+c \mathrm{e}^{\varphi(M)} T(M, \partial) \partial .
\end{aligned}
$$

These operators are defined on the whole polynomial space $\mathbb{C}[x]$, and by Theorem 5 they satisfy (1.1)-(1.3) on $\mathbb{C}[x]$. We can now, for any $f \in \mathbb{C}[x]$, define $A_{1}, A_{2}$ and $A_{3}$ by the equations

$$
\begin{aligned}
\left(A_{1} f\right)(x) & =x f(x), \quad\left(A_{3} f\right)(x)=c \mathrm{e}^{\varphi(M)} f(-x)=c \mathrm{e}^{\varphi(x)} f(-x), \\
\left(A_{2} f\right)(x) & =c E(\varphi(M))\left[\mathrm{e}^{\varphi(M)} \psi(M)-\frac{1}{2} \varphi^{\prime}(M)\right] f(-x)+c \mathrm{e}^{\varphi(M)} f^{\prime}(-x) \\
& =\frac{c}{\cosh (\varphi(x))}\left[\mathrm{e}^{\varphi(x)} \psi(x)-\frac{1}{2} \varphi^{\prime}(x)\right] f(-x)+c \mathrm{e}^{\varphi(x)} f^{\prime}(-x) .
\end{aligned}
$$

Example 2. Let $s$ be a positive odd integer and put $\varphi(A)=\alpha A$ and $\psi(A)=\beta A^{s}$, where $\alpha, \beta \in \mathbb{C}$, in the general solution given by Theorem 5 . As in Example 1 we consider the operators $A=\partial$ and $B=M$ defined on the linear space $\mathbb{C}[x]$. Then we have

$$
\begin{aligned}
& A_{1}=\partial, \quad A_{3}=c T(M, \partial) \mathrm{e}^{\alpha \partial}, \\
& A_{2}=c T(M, \partial) E(\alpha \partial)\left[\mathrm{e}^{\alpha \partial} \beta \partial^{s}-\frac{\alpha}{2}\right]+c M T(M, \partial) \mathrm{e}^{\alpha \partial} .
\end{aligned}
$$

We can now define $A_{1}, A_{2}$ and $A_{3}$ on the polynomial space $\mathbb{C}[x]$ by the equations

$$
\begin{aligned}
\left(A_{1} f\right)(x) & =f^{\prime}(x), \quad\left(A_{3} f\right)(x)=c T(M, \partial) \mathrm{e}^{\alpha \partial} f(x)=c f(-x+\alpha), \\
\left(A_{2} f\right)(x) & =c T(M, \partial) E(\alpha \partial)\left[\beta f^{(s)}(x+\alpha)-\frac{\alpha}{2} f(x)\right]+c x f(-x+\alpha) \\
& =c T(M, \partial) \sum_{n=0}^{\infty} \frac{E_{2 n} \alpha^{2 n}}{(2 n) !}\left[\beta f^{(s+2 n)}(x+\alpha)-\frac{\alpha}{2} f^{(2 n)}(x)\right]+c x f(-x+\alpha) \\
& =c \sum_{n=0}^{\infty} \frac{E_{2 n} \alpha^{2 n}}{(2 n) !}\left[\beta f^{(s+2 n)}(-x+\alpha)-\frac{\alpha}{2} f^{(2 n)}(-x)\right]+c x f(-x+\alpha) .
\end{aligned}
$$


These operators are defined on the whole polynomial space $\mathbb{C}[x]$, and by Theorem 5 they satisfy relations (1.1)-(1.3) on $\mathbb{C}[x]$. By a simple computation we have

$$
\begin{aligned}
& \left(A_{1} A_{2}+A_{2} A_{1}\right) f(x)=c f(-x+\alpha)=\left(A_{3} f\right)(x), \\
& \left(A_{1} A_{3}+A_{3} A_{1}\right) f(x)=-c f^{\prime}(-x+\alpha)+c f^{\prime}(-x+\alpha)=0 .
\end{aligned}
$$

Moreover,

$$
\begin{aligned}
& \left(A_{2} A_{3} f\right)(x)=c \sum_{n=0}^{\infty} \frac{E_{2 n} \alpha^{2 n}}{(2 n) !}\left[c \beta(-1)^{s} f^{(s+2 n)}(x)-\frac{1}{2} c \alpha f^{(2 n)}(x-\alpha)\right]+c^{2} x f(x), \\
& \left(A_{3} A_{2} f\right)(x)=c^{2} \sum_{n=0}^{\infty} \frac{E_{2 n} \alpha^{2 n}}{(2 n) !}\left[\beta f^{(s+2 n)}(x)-\frac{\alpha}{2} f^{(2 n)}(x+\alpha)\right]+c^{2}(-x+\alpha) f(x),
\end{aligned}
$$

showing that

$$
\left(A_{2} A_{3}+A_{3} A_{2}\right) f(x)=\alpha c^{2} f(x)-\frac{1}{2} c^{2} \sum_{n=0}^{\infty} \frac{E_{2 n}}{(2 n) !} \alpha^{2 n+1}\left[f^{(2 n)}(x-\alpha)+f^{(2 n)}(x+\alpha)\right] .
$$

Thus, the relation $\left(A_{2} A_{3}+A_{3} A_{2}\right) f(x)=0$ is satisfied if and only if the function $f$ satisfies

$$
f(x)=\frac{1}{2} \sum_{n=0}^{\infty} \frac{E_{2 n}}{(2 n) !} \alpha^{2 n}\left[f^{(2 n)}(x-\alpha)+f^{(2 n)}(x+\alpha)\right] .
$$

The relations (1.1)-(1.3) hold on $\mathbb{C}[x]$ and so equation (3.2) holds for $f \in \mathbb{C}[x]$.

Example 3. Let in Example 2 the constant $c=1$ and the parameters $\alpha=\beta=0$. Then we obtain

$$
A_{1}=\partial, \quad A_{2}=M T(M, \partial), \quad A_{3}=T(M, \partial) .
$$

In this case we have the simple relation $A_{2}=M A_{3}$ and for any polynomial $p(x) \in \mathbb{C}[x]$, we obtain

$$
\left(A_{1} p\right)(x)=p^{\prime}(x), \quad\left(A_{2} p\right)(x)=x p(-x), \quad\left(A_{3} p\right)(x)=p(-x) .
$$

These three operators can be defined for any differentiable function $f$ and they satisfy the commutation relations (1.1)-(1.3), since one can easily verify that

$$
\begin{aligned}
& \left(A_{1} A_{2}+A_{2} A_{1}\right) f(x)=f(-x)=A_{3} f(x), \\
& \left(A_{1} A_{3}+A_{3} A_{1}\right) f(x)=-f^{\prime}(-x)+f^{\prime}(-x)=0, \\
& \left(A_{2} A_{3}+A_{3} A_{2}\right) f(x)=x f(x)-x f(x)=0 .
\end{aligned}
$$

Example 4. Let us have a closer look at the operators studied in Example 2 in the special case where $c=\alpha=1$ and $\beta=0$, namely

$$
A_{1}=\partial, \quad A_{2}=M T(M, \partial) \mathrm{e}^{\partial}-\frac{1}{2} T(M, \partial) E(\partial), \quad A_{3}=T(M, \partial) \mathrm{e}^{\partial} .
$$


We can express $E(\partial)$ in terms of the generating function for the Euler numbers, obtaining

$$
\begin{aligned}
E(\partial) & =2 \mathrm{e}^{\partial}\left(\mathrm{e}^{2 \partial}+1\right)^{-1}=\mathrm{e}^{\partial}\left(\frac{1}{2}+\frac{1}{2} \mathrm{e}^{2 \partial}\right)^{-1}=\mathrm{e}^{\partial}\left(1+\frac{1}{2}\left(\mathrm{e}^{2 \partial}-1\right)\right)^{-1} \\
& =\mathrm{e}^{\partial} \sum_{k=0}^{\infty} \frac{(-1)^{k}}{2^{k}}\left(\mathrm{e}^{2 \partial}-1\right)^{\mathrm{k}}=\mathrm{e}^{\partial} \sum_{\mathrm{k}=0}^{\infty} \frac{(-1)^{\mathrm{k}}}{2^{\mathrm{k}}} \sum_{\mathrm{l}=0}^{\mathrm{k}}\left(\begin{array}{l}
\mathrm{k} \\
1
\end{array}\right) \mathrm{e}^{2(\mathrm{k}-1) \partial}(-1)^{\mathrm{l}} \\
& =\sum_{k=0}^{\infty} \frac{(-1)^{k}}{2^{k}} \sum_{l=0}^{k}(-1)^{l}\left(\begin{array}{l}
k \\
l
\end{array}\right) \mathrm{e}^{(2(k-l)+1) \partial} .
\end{aligned}
$$

By virtue of the equality $\mathrm{e}^{n \partial} f(x)=f(x+n)$ and equation (3.1), it is now reasonable to define

$$
\begin{aligned}
& \left(A_{1} f\right)(x)=f^{\prime}(x), \quad\left(A_{3} f\right)(x)=f(1-x), \\
& \left(A_{2} f\right)(x)=x f(1-x)-\sum_{k=0}^{\infty} \frac{(-1)^{k}}{2^{k+1}} \sum_{l=0}^{k}(-1)^{l}\left(\begin{array}{l}
k \\
l
\end{array}\right) f(2(k-l)+1-x),
\end{aligned}
$$

where $f \in \mathbb{C}[x]$. In order to verify the commutation relations, we compute

$$
\begin{aligned}
& A_{1} A_{2} f(x)=f(1-x)-x f^{\prime}(1-x)+\sum_{k=0}^{\infty} \sum_{l=0}^{k} \frac{(-1)^{k+l}}{2^{k+1}}\left(\begin{array}{c}
k \\
l
\end{array}\right) f^{\prime}(2(k-l)+1-x), \\
& A_{2} A_{1} f(x)=x f^{\prime}(1-x)+\sum_{k=0}^{\infty} \sum_{l=0}^{k} \frac{(-1)^{k+l}}{2^{k+1}}\left(\begin{array}{c}
k \\
l
\end{array}\right) f^{\prime}(2(k-l)+1-x) .
\end{aligned}
$$

It follows that

$$
\begin{aligned}
& \left(A_{1} A_{2}+A_{2} A_{1}\right) f(x)=f(1-x)=A_{3} f(x), \\
& \left(A_{1} A_{3}+A_{3} A_{1}\right) f(x)=-f^{\prime}(1-x)+f^{\prime}(1-x)=0 .
\end{aligned}
$$

Moreover,

$$
\begin{aligned}
& A_{2} A_{3} f(x)=A_{2} f(1-x)=x f(x)-\sum_{k=0}^{\infty} \sum_{l=0}^{k} \frac{(-1)^{k+l}}{2^{k+1}}\left(\begin{array}{c}
k \\
l
\end{array}\right) f(x-2(k-l)), \\
& A_{3} A_{2} f(x)=(1-x) f(x)-\sum_{k=0}^{\infty} \sum_{l=0}^{k} \frac{(-1)^{k+l}}{2^{k+1}}\left(\begin{array}{c}
k \\
l
\end{array}\right) f(x+2(k-l)),
\end{aligned}
$$

and hence

$$
\left(A_{2} A_{3}+A_{3} A_{2}\right) f(x)=f(x)-\sum_{k=0}^{\infty} \sum_{l=0}^{k} \frac{(-1)^{k+l}}{2^{k+1}}\left(\begin{array}{l}
k \\
l
\end{array}\right)[f(x-2(k-l))+f(x+2(k-l))] .
$$

The relation $\left(A_{2} A_{3}+A_{3} A_{2}\right) f(x)=0$ is satisfied if and only if

$$
f(x)=\sum_{k=0}^{\infty} \frac{(-1)^{k}}{2^{k+1}} \sum_{l=0}^{k}(-1)^{l}\left(\begin{array}{l}
k \\
l
\end{array}\right)[f(x-2(k-l))+f(x+2(k-l))] .
$$


By Example 2 and Example 4 we have a proof of the following statement:

Proposition 6. Every polynomial $p(x) \in \mathbb{C}[x]$ satisfies the relations

(a) $p(x)=\frac{1}{2} \sum_{k=0}^{\infty} \frac{E_{2 k}}{(2 k) !} \alpha^{2 k}\left[p^{(2 k)}(x-\alpha)+p^{(2 k)}(x+\alpha)\right]$,
(b) $p(x)=\sum_{k=0}^{\infty} \frac{(-1)^{k}}{2^{k+1}} \sum_{l=0}^{k}(-1)^{l}\left(\begin{array}{c}k \\ l\end{array}\right)[p(x-2(k-l))+p(x+2(k-l))]$.

Example 5. Consider the differentiation and multiplication operators $\partial$ and $M$ introduced at the beginning of this section. The linear transformation

$$
a=\frac{x_{0}}{\sqrt{2}} \partial+\frac{1}{\sqrt{2} x_{0}} M, \quad a^{*}=-\frac{x_{0}}{\sqrt{2}} \partial+\frac{1}{\sqrt{2} x_{0}} M,
$$

where $x_{0}$ is a positive real constant, gives a couple of operators $a$ and $a^{*}$ satisfying the canonical commutation relation $a a^{*}-a^{*} a=1$. This follows from the fact that they are obtained from $\partial$ and $M$ by an $S L(2, \mathbb{C})$-transformation. Now let $\left\{\phi_{\nu}\right\}_{\nu=0}^{\infty}$ be a sequence of functions defined by

$$
\sqrt{\nu+1} \phi_{\nu+1}(x)=a^{*} \phi_{\nu}(x), \quad \nu \geq 0 ; \quad \phi_{0}(x)=\frac{1}{\sqrt{\sqrt{\pi} x_{0}}} \exp \left(-\frac{1}{2}\left(\frac{x}{x_{0}}\right)^{2}\right) .
$$

It follows that

$$
\begin{aligned}
\phi_{n}(x) & =\frac{1}{\sqrt{n !}}\left(a^{*}\right)^{n} \phi_{0}(x)=\frac{1}{\sqrt{n ! \sqrt{\pi} x_{0}}}\left(a^{*}\right)^{n} \exp \left(-\frac{1}{2}\left(\frac{x}{x_{0}}\right)^{2}\right) \\
& =\frac{1}{\sqrt{2^{n} n ! \sqrt{\pi} x_{0}}} \exp \left(-\frac{1}{2}\left(\frac{x}{x_{0}}\right)^{2}\right) H_{n}\left(\frac{x}{x_{0}}\right),
\end{aligned}
$$

with $n=0,1,2, \ldots$, where $H_{n}$ are the Hermite polynomials. It can be shown that $a \phi_{0}=0$ and $a \phi_{\nu}=\sqrt{\nu} \phi_{\nu-1}$ for $\nu \geq 1$. Furthermore,

$$
\left(a^{*}\right)^{k} a^{k} \phi_{\nu}= \begin{cases}\frac{\nu !}{(\nu-k) !} \phi_{\nu} & \text { if } k \leq \nu \\ 0 & \text { if } k>\nu,\end{cases}
$$

for any $k \in \mathbb{N}$. The sequence of functions $\left\{\phi_{\nu}\right\}_{\nu=0}^{\infty}$ describes the energy eigenstates of the simple quantum mechanical harmonic oscillator. Consider now the two differential operators $a^{*}$ ("creation" operator) and $a$ ("annihilation" operator) defined on the linear space $\Omega=\operatorname{linspan}_{\mathbb{C}}\left(\left\{\phi_{\nu}\right\}_{\nu=0}^{\infty}\right)$ consisting of all complex linear combinations of functions from the set of eigenfunctions $\left\{\phi_{\nu}\right\}_{\nu=0}^{\infty}$. Since now $a a^{*}-a^{*} a=1$, we can replace $\partial$ and $M$ in the representation studied in Example 2 by $a$ and $a^{*}$, respectively, thus obtaining (with $c=\alpha=1$ and $\beta=0$ )

$$
A_{1}=a, \quad A_{2}=a^{*} T\left(a^{*}, a\right) e^{a}-\frac{1}{2} T\left(a^{*}, a\right) E(a), \quad A_{3}=T\left(a^{*}, a\right) e^{a} .
$$

Acting on an eigenfunction $\phi_{\nu}$ we have by a straightforward computation $T\left(a^{*}, a\right) \phi_{\nu}=$ $(-1)^{\nu} \phi_{\nu}$. Moreover, knowing that $a \phi_{\nu}=\sqrt{\nu} \delta_{0 \nu} \phi_{\nu-1}$ where $\delta_{0 \nu}$ is the Kronecker symbol, 
it follows that for all $\nu \geq 0$

$$
\begin{aligned}
e^{a} \phi_{\nu} & =\sum_{k=0}^{\nu} \sqrt{\frac{1}{k !}\left(\begin{array}{l}
\nu \\
k
\end{array}\right)} \phi_{\nu-k}=\sum_{j=0}^{\nu} \sqrt{\frac{1}{(\nu-j) !}\left(\begin{array}{c}
\nu \\
j
\end{array}\right)} \phi_{j}, \\
E(a) \phi_{\nu} & =\sum_{k=0}^{\infty} \frac{E_{2 k}}{(2 k) !} a^{2 k} \phi_{\nu}=\sum_{k=0}^{\left[\frac{\nu}{2}\right]} E_{2 k} \sqrt{\frac{1}{(2 k) !}\left(\begin{array}{c}
\nu \\
\nu-2 k
\end{array}\right)} \phi_{\nu-2 k} .
\end{aligned}
$$

Hence,

$$
\begin{aligned}
& A_{1} \phi_{\nu}=\sqrt{\nu} \phi_{\nu-1}, \quad \nu \geq 1, \quad A_{1} \phi_{0}=0 \\
& A_{2} \phi_{\nu}=\sum_{j=0}^{\nu}(-1)^{j} \sqrt{\frac{j+1}{(\nu-j) !}\left(\begin{array}{c}
\nu \\
j
\end{array}\right)} \phi_{j+1}-\frac{(-1)^{\nu}}{2} \sum_{j=0}^{\left[\frac{\nu}{2}\right]} E_{2 j} \sqrt{\frac{1}{(2 j) !}\left(\begin{array}{c}
\nu \\
\nu-2 j
\end{array}\right)} \phi_{\nu-2 j}, \\
& A_{3} \phi_{\nu}=\sum_{j=0}^{\nu}(-1)^{j} \sqrt{\frac{1}{(\nu-j) !}\left(\begin{array}{c}
\nu \\
j
\end{array}\right)} \phi_{j} .
\end{aligned}
$$

\section{Appendix}

Recall that a $\mathbb{Z}_{2}^{n}$-graded (colour) generalized Lie algebra is a $\mathbb{Z}_{2}^{n}$-graded linear space

$$
X=\bigoplus_{\gamma \in \mathbb{Z}_{2}^{n}} X_{\gamma}
$$

with a bilinear multiplication (bracket) $\langle\cdot, \cdot\rangle: X \times X \rightarrow X$ obeying:

Grading axiom: $\left\langle X_{\alpha}, X_{\beta}\right\rangle \subseteq X_{\alpha+\beta}$.

Graded skew-symmetry: $\langle a, b\rangle=-(-1)^{\alpha \cdot \beta}\langle b, a\rangle$.

Generalized Jacobi identity:

$$
(-1)^{\alpha \cdot \gamma}\langle a,\langle b, c\rangle\rangle+(-1)^{\gamma \cdot \beta}\langle c,\langle a, b\rangle\rangle+(-1)^{\beta \cdot \alpha}\langle b,\langle c, a\rangle\rangle=0
$$

for all $\alpha=\left(\alpha_{1}, \ldots, \alpha_{n}\right), \beta=\left(\beta_{1}, \ldots, \beta_{n}\right), \gamma=\left(\gamma_{1}, \ldots, \gamma_{n}\right)$ in $\mathbb{Z}_{2}^{n}$, and $a \in X_{\alpha}, b \in X_{\beta}$, $c \in X_{\gamma}$, where $\alpha \cdot \beta=\sum_{i=1}^{n} \alpha_{i} \beta_{i}$ etc., with $\sum$ meaning addition in $\mathbb{Z}_{2}$. The elements of $\bigcup_{\gamma \in \mathbb{Z}_{2}^{n}} X_{\gamma}$ are called homogeneous.

Any $\mathbb{Z}_{2}^{n}$-graded generalized Lie algebra $X$ can be embedded in its universal enveloping algebra $U(X)$ in such a way that, for homogeneous $a \in X_{\alpha}$ and $b \in X_{\beta}$, the bracket $\langle\cdot, \cdot\rangle$ becomes a commutator $[a, b]=a b-b a$ when $\alpha \cdot \beta$ is even, or an anticommutator $\{a, b\}=a b+b a$ when $\alpha \cdot \beta$ is odd [14].

Now take $X$ to be a $\mathbb{Z}_{2}^{3}$-graded linear space

$$
X=X_{(1,1,0)} \oplus X_{(1,0,1)} \oplus X_{(0,1,1)}
$$

with the homogeneous basis $A_{1} \in X_{(1,1,0)}, A_{2} \in X_{(1,0,1)}, A_{3} \in X_{(0,1,1)}$. The homogeneous components graded by the elements of $\mathbb{Z}_{2}^{3}$ different from $(1,1,0),(1,0,1)$ and $(0,1,1)$ are 
zero and so are omitted. If the $\mathbb{Z}_{2}^{3}$-graded bilinear multiplication $\langle\cdot, \cdot\rangle$ turns $X$ into a $\mathbb{Z}_{2}^{3}$-graded generalized Lie algebra, then $\left\langle A_{i}, A_{i}\right\rangle=0, i=1,2,3$ and

$$
\left\langle A_{1}, A_{2}\right\rangle=c_{12} A_{3}, \quad\left\langle A_{2}, A_{3}\right\rangle=c_{23} A_{1}, \quad\left\langle A_{3}, A_{1}\right\rangle=c_{31} A_{2} .
$$

When $a$ and $b$ are in different homogeneous subspaces, it follows that $\langle a, b\rangle=\langle b, a\rangle$, whereas $\langle a, b\rangle=-\langle b, a\rangle$ if $a$ and $b$ belong to the same one. Moreover, the generalized Jacobi identity is valid. Now put $c_{12}=1, c_{23}=0$ and $c_{31}=0$. The algebra $X$ so defined has as its universal enveloping algebra the colour Heisenberg Lie algebra.

\section{Acknowledgements}

The authors would like to thank Professor Jouko Mickelsson, Dr. Lars Hellström and Dr. Edwin Langmann for useful comments. The first author is grateful to the Centre for Mathematical Sciences, Lund University for hospitality during his visits in Lund. This work has been partially supported by the Crafoord Foundation, the Royal Physiographic Society in Lund, the Swedish Royal Academy of Sciences and the Swedish Foundation for International Cooperation in Research and Higher Education (STINT).

\section{References}

[1] Bahturin Yu, Basic Structures of Modern Algebra, Kluwer Academic Publishers, Dordrecht, 1993.

[2] Bahturin Yu A, Mikhalev A A, Petrogradsky V M and Zaicev M V, Infinite Dimensional Lie Superalgebras, Walter de Gruyter, Berlin, 1992.

[3] Green H S and Jarvis P D, Casimir invariants, characteristic identities and Young diagrams for Colour algebras and superalgebras, J. Math. Phys. 24 (1983), 1681-1687.

[4] Hellström L, The Diamond Lemma for Power Series Algebras, Doctoral Thesis, no 23, 2002, Umeå University.

[5] Hellström L and Silvestrov S D, Commuting Elements in q-Deformed Heisenberg Algebras, World Scientific, 2000, 256 pp.

[6] Kac V G, Lie Superalgebras, Adv. Math. 26 (1977), 8-96.

[7] Kleeman R, Commutation factors on generalized Lie algebras, J. Math. Phys. 26 (1985), 2405-2412.

[8] Kwasniewski A K, Clifford- and Grassmann-like algebras - Old and new, J. Math. Phys. 26 (1985), 2234-2238.

[9] Marcinek W, Generalized Lie algebras and Related Topics,1,2, Acta Univ. Wratislaviensis ( Matematyka, Fizyka, Astronomia ) LV, 1170 (1991), $3-52$.

[10] Ostrovskii V L and Silvestrov S D, Representations of the real forms of the graded analogue of the Lie algebra sl(2, C), Ukrain. Mat. Zh. 44 (1992), 1518-1524; (English translation: Ukrainian Math. J. 44 (1993), 1395-1401). 
[11] Persson L, Silvestrov S D and Strunk P, Central elements of the second order in three-dimensional generalised Lie algebras, Czech. J. Phys. 47 (1997), 99-106.

[12] Putnam C R, Commutation Properties of Hilbert Space Operators and Related Topics, Springer-Verlag, Berlin Heidelberg, 1967.

[13] Rittenberg V and Wyler D, Generalized Superalgebras, Nucl. Phys. B 139 (1978), 189-202.

[14] Scheunert M, Generalized Lie algebras, J. Math. Phys. 20 (1979), 712-720.

[15] Scheunert M, Graded tensor calculus, J. Math. Phys. 24 (1983), 2658-2670.

[16] Sigurdsson G and Silvestrov S D, Canonical involutions in three-dimensional generalised Lie algebras, Czech. J. Phys. 50 (2000), 181-186.

[17] Silvestrov S D, Hilbert space representations of the graded analogue of the Lie algebra of the group of plane motions, Studia Mathematica 117 (1996), 195-203.

[18] Silvestrov S D, Representations of Commutation Relations. A Dynamical Systems Approach, Hadronic Journal Supplement, 11 (1996), 1-116.

[19] Silvestrov S D, On the classification of 3-dimensional coloured Lie algebras, in "Quantum Groups and Quantum Spaces", Banach Center Publications 40 (1997), 159-170.

[20] Wielandt H, Über die Unbeschränkheit der Schrödingerschen Operatoren der Quanten-mechanik, Math. Ann. 121 (1949), 21.

[21] Wintner A, The unboundedness of quantum-mechanical matrices, Phys. Rev. 71 (1947), 738-739. 